\title{
Upgraded Service Oriented Architecture for Smart Injection Molding.
}

\author{
Babloo, Devendra Singh, Ajay Kumar sharma
}

\begin{abstract}
This paper investigates current advances towards Smart Injection Molding, and presents the idea of Smart Molds or Molds 4.0. It exhibits a contextual analysis of the assembling of a medicinal gadget, including the test set-up of an instrumented shape, just as general suggestions on the most proficient method to actualize a Smart Manufacturing vision in the plastic Industry 4.0. Moreover, it incorporates a proposition of an Advanced Cyber-Physical System (CPS) Service Oriented Architecture (SOA) for constant observing and information examination of a shrewd microinjection trim procedure and for brilliant molds instrumentation as an approach to acknowledge such savvy vision.
\end{abstract}

Keywords: Smart Injection Molding, Smart Molds, Molds 4.0, Cloud Manufacturing, Cyber-Physical System, Industrial Internet of Things, Service Oriented Architecture, Industry 4.0.

\section{INTRODUCTION}

Ongoing advances continuously checking and control of assembling forms, on account of sensor-information, propelled information investigation and PC preparing power, have supported the acknowledgment of Smart Manufacturing and Industry 4.0 dreams [1] [2]. This gives increasingly dependable approaches to help related issues like "advanced quality administration" [3] and "zerodeformity producing" [4]. In any case, constrained consideration has been placed into the advancement of Smart Injection Molding Processes notwithstanding its high assembling intricacy and its noteworthy commitment to the mechanical economy in the creation of significant worth included items [5]. At the shop-floor level, as per Kenig et al. [6], the ideal components in a clever control of infusion embellishment are: (an) a dependable, continuous, estimation of the procedure parameters, (b) a procedure model that portrays the connection between the procedure parameters and the part properties, and (c) training capacities of the control framework to guarantee that it can distinguish deviations from procedure cutoff points, and their impacts on the nature of the part. Consequently, Smart Injection Molding can be characterized as a detecting versatile controlled infusion trim procedure for delivering parts by infusing liquid material into a form pit in a constant observed and controlled generation condition, where the formed part will chill off, harden and hardness into the shape that has adjusted to the shape of the form depression.

Revised Manuscript Received on February 05, 2020.

* Correspondence Author

Mr. Babloo, M.Tech Student, Mechanical Department, Sachdeva institute of technology, Farah, Mathura, Uttar Pradesh, India.

Mr. Devendra Singh, Assistant Professor, Mechanical Department, Sachdeva institute of technology, Farah, Mathura, Uttar Pradesh, India.

Mr. Ajay Kumar Sharma, Assistant Professor, Mechanical Department, Institute of Engineering and technology, Lucknow, Uttar Pradesh, India

(C) The Authors. Published by Blue Eyes Intelligence Engineering and Sciences Publication (BEIESP). This is an open access article under the CC BY-NC-ND license (http://creativecommons.org/licenses/by-nc-nd/4.0/)
Such control factors may incorporate screw speed, shape weight, infusion temperature, and take-out-time. Besides, incorporated item process quality control in infusion forming towards zero-absconds requires in excess of a shrewd assembling process. It requires a Smart Mold, which can be portrayed as a sensorised shape with self-and procedure checking abilities supporting the advanced quality administration of formed parts and its own prescient and preventive upkeep (for example resource care) in light of the absolute control of weight, temperature, vibrations, cycles, uprightness, hot sprinter and cooling parameters of the shrewd item. Different prerequisites for Smart Molds and Smart Injection Molding include: (a) quicker handling of the formed part,

(b) finished parts out of the form requiring no further work,

(c) faster changeover of molds to oblige a more prominent assortment of shaped parts, (d) robotized materials taking care of and preparing ability, and (e) information social affair and capacity of value records for each part created from a particular form [7].

As to basics of Industry 4.0 vision, Molds 4.0 speak to a domain where infusion embellishment machines are viewed as independent hardware that can work together with other assembling gear by means of PC systems and with the venture data frameworks (for example MES) for dependable, quicker and more astute basic leadership. Such Smart Equipment is viewed as a 'specialist co-op entertainer' or 'detecting savvy operator' with the capacity to consistently interoperate with other and required assembling assets to all the more productively build up coherent groups to more readily adapt to the prerequisites of the assembling procedure worth chain. All the required data and control streams are incorporated and facilitated by a more extensive and multi-layered frameworks design dependent on the standards of Service Oriented Architecture (SOA) [8] and the advantages given by distributed computing and processing inescapability.

This empowers information organizing with other assembling data frameworks so as to help higher profitability and quality execution of the entire generation framework. A Smart Injection Molding Machine and a Smart Mold are then observed as empowering agents for a "Form Industry 4.0".

Finally, the exploratory set-up alludes to the improvement of an Advanced Cyber-Physical System (CPS) SOA Architecture for ongoing checking and information examination of a keen infusion trim procedure, and the instrumentation of its relating "Shrewd Mold" with piezoelectric sensors for information obtaining, both set-ups planning to attract general proposals terms of sensors innovation, information procurement framework, and sign molding. 


\section{Upgraded Service Oriented Architecture for Smart Injection Molding.}

\section{TOWARDSS SMARTS MOLDSS ORS MOLDSS 4.0}

Piezoelectric technologies have been introduced in several applications for acceleration, force and pressure sensing [9].

Furthermore, traditional devices used are mainly accelerometers, dynamometers and acoustic emission detectors, which have been used in thousands of research works in machine tools condition monitoring and other automation applications [9]. However, it is recognized that the recent introduction of temperature and pressure "piezoelectric sensors" for conditionbased monitoring could lead to fostering a Smart Injection Molding Process. In this particular area, it is important to test first the reliability of signals by performing additional validations with the aid of "Finite Element Analysis".

The work presented in this section is intended to exemplify the engineering behind the construction of sensorised molds as the first step of the construction of a test-bed for Molds 4.0. The selected case study was a mold adapted to hold four cavities and fabricated for tooling microinjectionmolding machines [10]. The plastic part to be injected is a locking ligation system, which is often used in surgical procedures for the treatment of several medical conditions and injuries. Fig. 1 shows the engineering design of the cavities, including runners, gates and the digital representation of the part to be injected. The characteristics of the Mold 4.0 can be seen in Fig. 2, in which the general geometric and functional features are the following: four cavities, sliders for enabling the injection in hidden areas, runners, ejection pins, gates and plates [10].
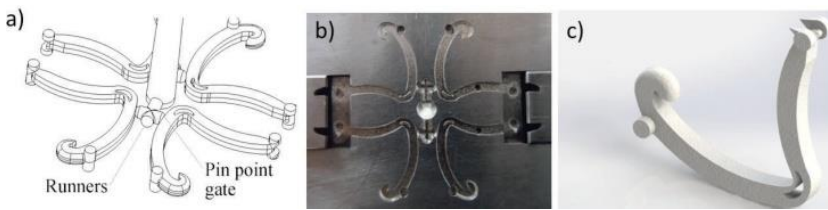

Fig. 1. (a) Design of Cavities for Microinjection Molding (b) Cavities and Sliders, and (c) Polymer Ligation System

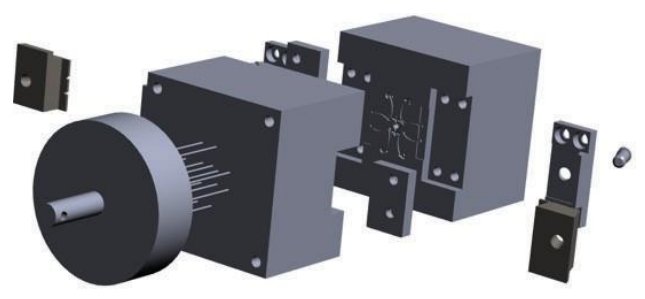

Fig.s 2.s Generals Featuress ofs as Molds fors Lockings Ligations Systemss Injections Molding

Once the mold design and fabrication were made, the next step is the selection of the variables to be monitored and the selection of the appropriate sensor system. It is acknowledged that pressure inside the cavity during the injection process is related with several quality and productivity indicators, but it is always correlated with the temperature distribution

due to the changes in rheological properties that are taking effect inside the cavities. Thus, by measuring real-time temperature and pressure, one can be focused in the most important variables to be controlled.

After the selection of critical variables, the next step is the installation of piezoelectric sensors inside the cavity. The selection of suitable sensors depends on the complexity of geometric elements of the part and on their adaptation to the small scales of these geometries. piezoelectric sensors selected for this application are showed in Table I.

Table I. Characteristics Of Piezoelectric SENSORS

\begin{tabular}{|c|c|c|c|}
\hline Range & Bar & $\begin{array}{c}0 \mathrm{~s}- \\
2000\end{array}$ \\
\hline Overload & Bar & 2500 \\
\hline Sensitivity & $\mathrm{pC} / \mathrm{bar}$ & $-4,891$ \\
\hline $\begin{array}{c}\text { Linearity, All } \\
\text { Range }\end{array}$ & $\% \mathrm{FSO}$ & $\begin{array}{c}<+/ \mathrm{s} \\
1\end{array}$ \\
\hline $\begin{array}{c}\text { Thermocouples } \\
\text { Types K }\end{array}$ & & $\begin{array}{c}\mathrm{NiCr}-\mathrm{s} \\
\mathrm{Ni}\end{array}$ \\
\hline $\begin{array}{c}\text { Operating } \\
\text { Temp.s Ranges } \\
\text { (general) }\end{array}$ & ${ }^{\circ} \mathrm{C}$ & $\begin{array}{c}0 \mathrm{~s}-\mathrm{s} \\
200\end{array}$ \\
\hline $\begin{array}{c}\text { Temp.s ats thes } \\
\text { Cavity }\end{array}$ & ${ }^{\circ} \mathrm{C}$ & $<\mathrm{s} 450$ \\
\hline
\end{tabular}

Afterwards, the signal conditioning is required to amplify pressure and temperature signals obtained from piezoelectric sensors. Two similar amplifiers are required for the system, one for the pressure signal and the other for temperature signal.

The injection molding process taken as reference is based on microinjection technologies, which are able to perform molding in small-scaled cavities and specialized for achieving higher precision levels than conventional processes. The machine used for exemplifying Smart Molding is a "Babyplast $\mathrm{t}^{1}$ " with the features showed in Table II.

Table II. Characteristics Of Microinjection Molding Machine

\begin{tabular}{|c|c|}
\hline Pistons diameter & $14 \mathrm{~s} \mathrm{~mm}$ \\
\hline Volumes (injected) & $9 \mathrm{~cm} 3$ \\
\hline Injections Pressure & $1340 \mathrm{~s} \mathrm{Kg} / \mathrm{cm} 2$ \\
\hline Clampings Force & $6.250 \mathrm{sg} / \mathrm{cm} 2 \mathrm{~s}(62.5 \mathrm{~s}$ \\
& $\mathrm{KN})$ \\
\hline Openings Force & $4 \mathrm{~s} \mathrm{KN}$ \\
\hline Openings Stroke & $30 \mathrm{~s}-\mathrm{s} 110 \mathrm{~s} \mathrm{~mm}$ \\
\hline Ejections Force & $5 \mathrm{~s} \mathrm{KN}$ \\
\hline Ejections Stroke & $45 \mathrm{~s} \mathrm{~mm}$ \\
\hline Hydraulics Pressure & $130 \mathrm{~s} \mathrm{Kg}$ \\
\hline Drys Cycle & $2.4 \mathrm{~s}$ \\
\hline Power & $2.9 \mathrm{~s} \mathrm{Kw}$ \\
\hline Weight & $120 \mathrm{~s} \mathrm{Kg}$ \\
\hline
\end{tabular}

The technical data of the data acquisition board selected are show in Table III. 
Table III. Characteristics Of Data Acquisition System

\begin{tabular}{|c|c|c|}
\hline Feature & Units & Value \\
\hline Measurings time & $\mathrm{s}$ & $<\mathrm{s} 600$ \\
\hline $\begin{array}{c}\text { Numbers ofs evaluations } \\
\text { objects }\end{array}$ & 48 \\
\hline $\begin{array}{c}\text { Numbers ofs reals times } \\
\text { threshold }\end{array}$ & $\begin{array}{c}\text { Pers } \\
\text { channel }\end{array}$ & Ups tos 2 \\
\hline Jitters real-times thresholds & $\mathrm{ms}$ & $<\mathrm{s} 0.18$ \\
\hline $\begin{array}{c}\text { Reactions times real-times } \\
\text { thresholds }\end{array}$ & $\mathrm{ms}$ & 4 \\
\hline Samplings rate & $\mathrm{Hz}$ & 1200 \\
\hline Cut-offs frequencys (3-dB) & $\mathrm{Hz}$ & 368 \\
\hline
\end{tabular}

The reference is "Kistler Como Injection basic type 2869b" with four channels (see Figure 3). The equipment and mold characteristics mentioned above were used in preliminary studies [10] to verify the accuracy of the parts, the capabilities of the process, and the reliability of the signals.

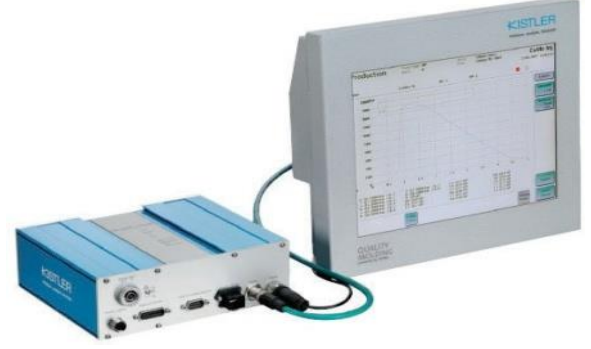

Fig. 3. Data Acquisition System - Kistler CoMo Injection

\section{SMARTS MOLDSS SENSORSS VALIDATION}

The capability to measure on-line temperature and pressure inside the cavity was tested in order to propose the instrumentation and data acquisition systems for further generalization under a Mold 4.0 vision.

The first outcome of this experimentation was the measurement of average pressure inside the cavity during the injection process. The results are shown in Fig. 4. They have been validated with Finite Element Analysis in a previous research work [10]. Industry 4.0, and how the concepts of Smart Injection Molding and Molds 4.0 may be hypothetically implemented in such ICT/OT infrastructure under the perspective of an Advanced Cyber-Physical System (CPS) Service Oriented Architecture (SOA).

Benefiting from the advances on Industrial Automation, ICTs and Control and Management models, shop-floor systems and equipment have turned into more active-entities within the wider, intensively collaborative, and smarter production environment that characterizes Industry 4.0 [11].

Smart Injection Molding Machines and Molds are then viewed as elements of a "cyber-physical production system" [11], being co-responsible for increasing the Smart Factory efficiency and sustainability while coping with the need of highly customized and shorter lifecycle products [13].

Classical Cyber-Physical Systems (CPS)'s architecture

is represented by integrated computational and physical capabilities, such as sensing, communication and actuation to physical world, with feedback loops where physical processes affect computations and vice-versa [11]. When immersed in the Industry 4.0 scenario Advanced CPS (A-CPS) Architectures [13] should incorporate the following 'design principles': Interoperability, Modularity, Digitalization/Virtualization, Real- Time Information, Service-Orientation, and Decentralization/ Autonomy [11] [12].

An Industry 4.0 scenario is however, a target to be reached by companies as they are mostly in the 'Industry 3.0' era yet. Therefore, it is important to support some "transition aspects" in their architectures so that A-CPS can also work within classical control models and legacy systems [14].

From the envisaged A-CPS point of view, this means that an industrial equipment (e.g. an injection molding machine) is

Number of Cycles Cavity 1 Average Pressure 9.979 [MPa]

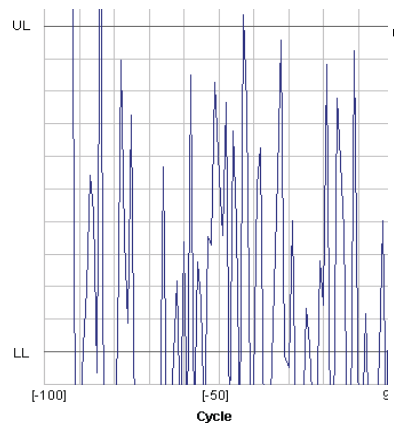

Number of Cycles Cavity 1 Average Pressure 9.979 [MPa]

Fig. 4. Pressure Measurements through the Injection Process using Piezoelectric Sensors

Regarding the temperature measurements, several plots have been obtained, showing consistent results with the obtained from the Finite Element Analysis. It is important to mention that the temperature profile showed the behavior in the outer part of the cavity, not in the melting surfaces due to the sensor location (see Figure 5).

Upper Limit $=48{ }^{\circ} \mathrm{C}$

Lower Limit $=38{ }^{\circ} \mathrm{C}$

UL

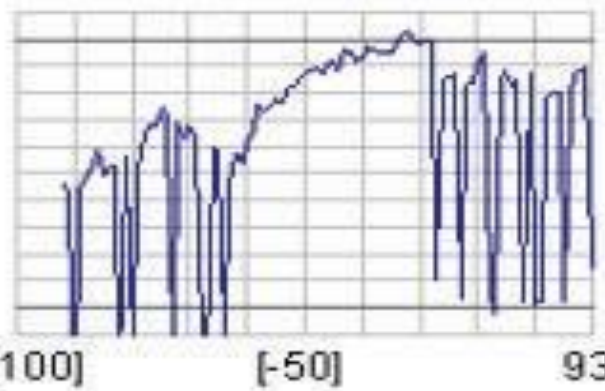

Cycle $[-100]$

[-50]

93

Fig. 5. Temperature Measurements through the Injection Process using Piezoelectric Sensors 


\section{PROPOSEDS ADVANCEDS CYBER-} PHYSICALS SYSTEMS SERVICES ORIENTEDS ARCHITECTURES FORS SMARTS INJECTIONS MOLDING

Once the concept of Smart Injection Molding was validated at the machine and mold levels, the next step should be to integrate this set-up into a Smart Factory infrastructure [11] [12]. This section is intended to present a proposal on how a Smart Manufacturing vision can be realized in the plastic a machine, its PLC, sensors and actuators, integrated via industrial networks, able to communicate with SCADA systems, and designed to manufacture (predefined) passive parts based on given (predefined) process plans. Instead, it is seen as an autonomous entity immersed in the Smart Factory's ecosystem embedded with production management and self- management abilities, including lean manufacturing concerns

[15] and eco-awareness [16]. It pro-actively proposes alternative process plans or refines them as production goes on and problems take place. A-CPS opportunistically competes for new orders based on its current and foreseen occupation and selfmanagement goals, turning scheduling and dispatching as emerging, bottom-up and adaptive plans. It is flexible for dealing with several different 'active' parts - intelligent objects

- [17] embedded with e.g. e-tags, and for autonomously interacting with other A-CPSs, manufacturing resources and computing systems (as MES and Cloud) aiming at looking for needed shopfloor partnerships to cope with current order's requirements, both in normal operation and exception handling. This takes advantage of creating temporary virtual production modules over existing physical layouts [as in 18], being all this executed under a Plan-Do-Check-Act / Adjust cycle. Figs. 6 and 7 show the general architecture and view of the envisioned A-CPS.

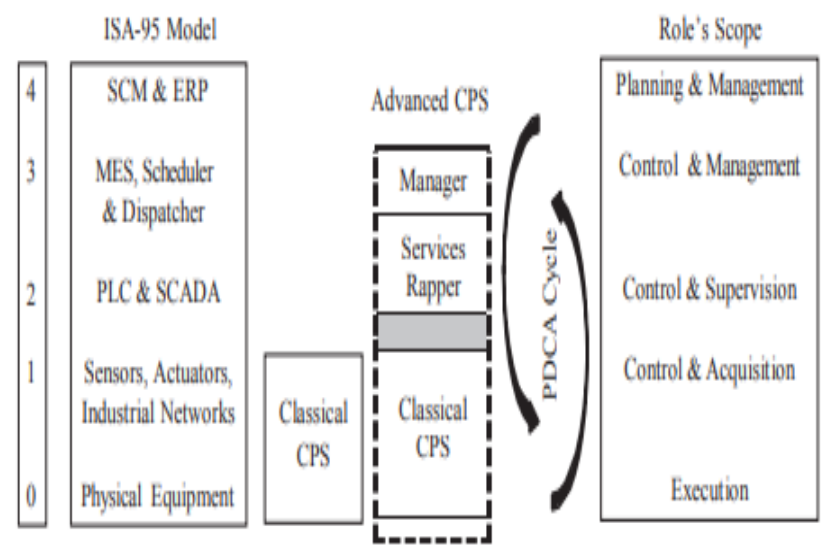

Fig. 6. Advanced CPS General View and Architecture

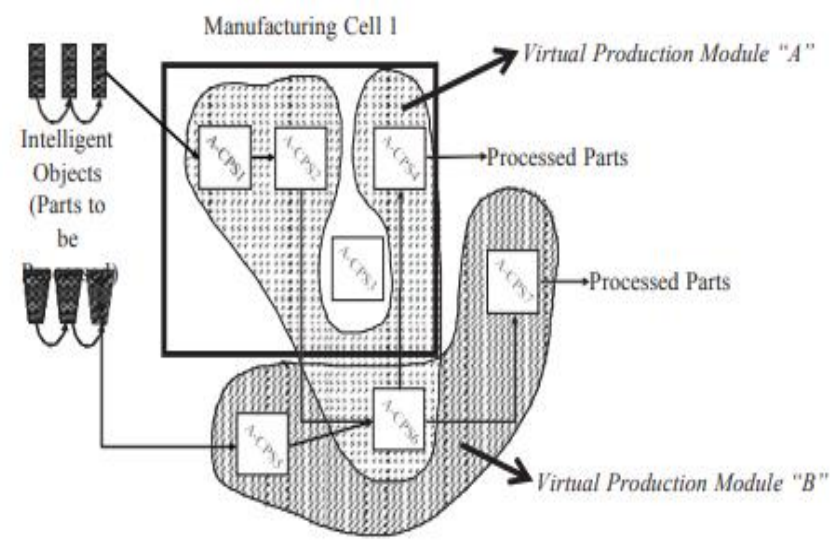

Fig. 7. Advanced CPSs as means to Leverage

Reconfigurable Production and Modularization

In order to respond to the Industry 4.0 requirements, mainly in terms of autonomy, decentralization, and modularity [13], the A-CPS Architecture extends the 'classical' CPS Architecture with two additional layers: (a) the Manager, and (b) the Services Wrapper (see Fig. 8). This value-added extension can be seen as a 'manufacturing connector'. This 'tandem architecture"

[19] is suitable for this case, allowing logical and physical decoupling of the planning / master / intelligence layer from the control / server / execution layer, but transparently to A-CPS client applications. In general, this model is not new at all. However, it adapts previous approaches - in that time taken as state-of-the-art and developed as isolated works - to the requirements of Industry 4.0, and make a sort of new IT approaches to converge, interoperate and work together so as to generate higher added value as well as to the try to better exploit the full potential of Industry 4.0 concept.

While the Manager works for satisfying the client applications' needs (e.g. in terms of real-time time information from the injection molding machine) and to handle the machine 'agenda' respecting local and global performance goals (for example, to collaborate with other A-CPS, to maximize its local utilization, and to minimize energy consumption as part of a global energy policy), the Server (i.e. the smart molding machine) keeps operating accordingly. This separation provides:

better performance of the involved computing processes as each layer works separately, (b) independence of implementation technologies used in the two levels and hence lower technology lock-in, (c) other client application (e.g. SCADA) can keep accessing the machine's PLC directly, and (d) it preserves the consolidated client-server communication mechanisms for which software development environments are mostly prepared for [18] [19]. 


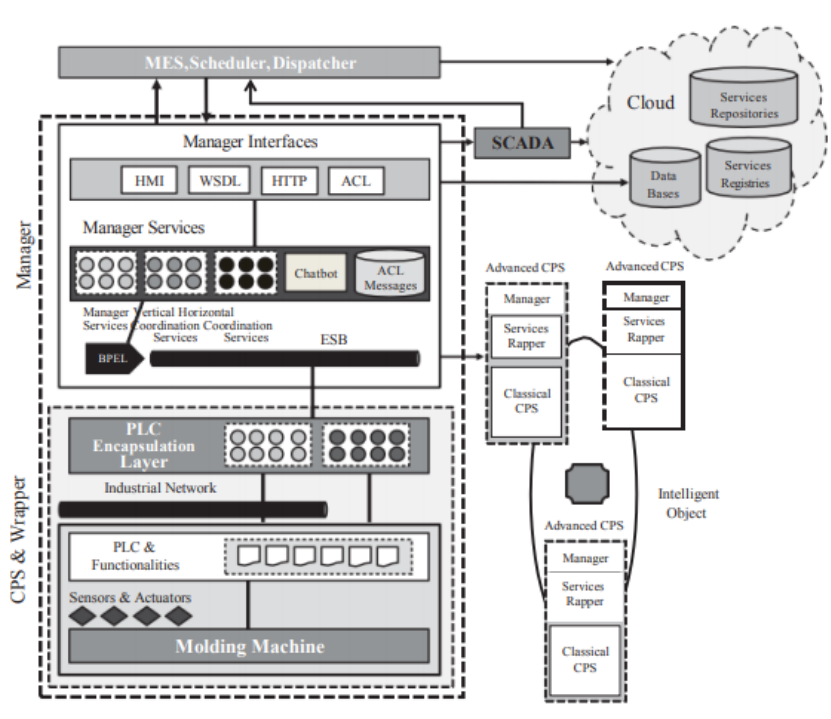

Fig. 8. Advanced CPS Detailed View and Architecture

The Wrapping [20] corresponds to an integration strategy / model and has three fundamental objectives: (a) acting as a layer to hide the heterogeneity and/or complexity of native implementation environments of the provider (the CPS server),

(a) providing a more homogeneous and/or standard way to access the server's functionalities from clients hence mitigating interoperability problems, and (c) creating new or aggregated views from the existing server's functionalities to clients [20].

The view of autonomous, self-evolving, adaptive, scalable, collaborative and flexible production entities is not new at all too, being quite explored in the 90's, mainly in the area of Holonic Manufacturing Systems (HMS) [21]. Such systems are composed of elementary entities called holons, which can form holarchies (e.g. a Virtual Production Module) - to attend the production requirements in place. Regarding to the inherent properties of multi-agent systems, agents have been largely used to model and implement HMS [21] or intelligent distributed manufacturing systems [22]. The Wrapping of a manufacturing resource (or other system) by agents is usually called as 'agentification' [18]. In summary, the Manager Layer of a A-CPS acts as an agent, which is a base to support intelligent, distributed and decentralized control [22] [23].

The Manager 'personalizes' the CPS within the production system, and represents the autonomy and decentralized decision-making properties of the CPS in the Industry 4.0 scenario [13]. In general, it allows: (a) the respective CPS to be 'plugged' into the global control architecture; to 'play' within it when required (as information and service providers, and to interact with other A-CPS), and (b) to be 'unplugged' from current production modules (see Fig. 6).

Depending on the physical organization of the shop floor and existing PLCs, a Manager can represent more than one CPS (e.g. a workstation composed of a smart mold machine equipped with an automated buffer and a robot to feed it).

The Manager has as main functions: (a) access/send

data from/to the CPS, (b) generates logs and reports (e.g. general and lean manufacturing-related performance indicators, energy consumption, etc.), (c) sends real-time information to client applications (e.g. MES, ERP, Cloud, and to other A-CPSs)

about the intelligent objects in place as well as from the PLC itself, (d) receives requests from them (about e.g. machine status, injection status, operational orders, replenishment proposals) including from the web, and (e) planning and coordination of both vertical "classical" hierarchical interactions (with e.g. MES and SCADA) and decentralized horizontal interactions (with e.g. other CPSs and the human interaction via the A- CPS's chatbot [24]).

There is a proper computing interface for each of these 'actors'. Human-Machine Interface (HMI) allows end-users to interact with the CPS using a local desktop interface. HTTP interface allows web, mobile and remote access to CPSs, including its chatbot's interface [25]. The Agent Communication Language (ACL) supports the interaction with other A-CPS, using proper protocols, messages and ontologies [25], http and web services [26] [27]. From the MAP/MMS ISO 9506 standard point of view, the manager and part of the CPS services can be seen as a VMD (Virtual Manufacturing Device) [28], bridging the A-CPS, other client systems (e.g. MES) and A-CPSs using standard-based manufacturing messages. These messages can then be embedded into the ACL and SOAP protocol [26] [27].

The Manager's functionalities are modelled as software services [8], which are accessed via their interfaces (e.g. via WDSL standard) [8] [26]. There are five categories of functionalities expressed as services: (a) the ones related to the Manager itself, (b) the ones to support the coordination messages and actions between the Manager and "vertical" client applications (e.g. MES), (c) the ones to support the coordination messages and actions between the Manager and "horizontal" client applications (i.e. other A-CPS), (d) the ones representing the chatbot's functionalities [24], and (e) the ones representing the agent's functionalities [26]. Following the same integration strategy, the PLC functionalities are also wrapped, in the PLC encapsulation layer. It can be seen as the PLC's high level "API". This encapsulation can comprise two types of access: (a) the ones that allow a communication with the native PLC functionalities, wrapped as services, and (b) the ones that allow a communication with eventual commercial products deployed on top of PLCs, via their API, to access data from the equipment, usually using the OPC-UA standard protocol.

Regarding that several interactions between the ACPS and the outer environment actually refer to information and actions upon the respective PLC and machine, the Manager's services should provide means to communicate with them. 


\section{Upgraded Service Oriented Architecture for Smart Injection Molding.}

However, the Manager and the CPS are decoupled computing environments, and the involved functionalities are usually implemented in different technologies. In order to overcome interoperability problems a BPEL (Business Process Execution Language) - ESB (Enterprise Service Bus) approach can be used [8] [22]. Although the communication between the Manager and the PLC tends to do not involve complex business processes models, a BPEL file can easily comprise the set of required services invocations in a standard way. It can feed the internal ESB, which acts as an interoperable bus binding given services invocations to the involved and heterogeneous wrapped PLC functionalities, considering their local implementation model and technologies.

All the involved services are registered in the 'Services Registries' and stored in the 'Services Repositories'. Many different deployment models can be adopted to support the whole system' architecture, be them totally deployed in a cloud, be them totally deployed in the company's local servers.

\section{EXPERIMENTAL RESULTS}

\section{Experiment Test Environment Settings}

When the injection molding machine started to process, large scale data packets poured into the database and was retrieved by our retrieval agents. The information retrieval agent was installed on the data collection server. In order to continuously retrieve the packets without missing data and errors, we further designed an intelligent agent socket packet linking mechanism, as discussed in Section 5.2. The test server simulation environment specifications for the information retrieval agent are listed in Table9.

The system was implemented with MS visual studio 2012 C\# programming language. All the production information was saved into an MS SQL server 2012. An Intel E5-2620 CPU with 8G RAM was adapted to simulate the agent retrieval environment. The injection molding machine manufacturing monitoring system interface is shown in Fig. 9. Each agent has its own agent ID. The injection molding machine manufacturing monitoring system is able to manage multiagents by creating new agents through a specific generator button called "Create exe". A delete function is also provided to purge created agents by clicking button "Delete exe".

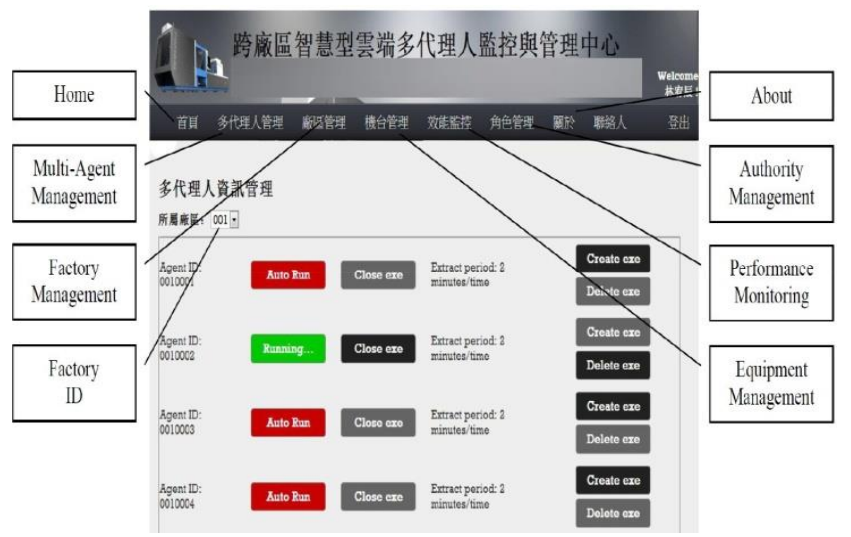

Fig. 9. Injection molding machine manufacturing monitoring system interface.
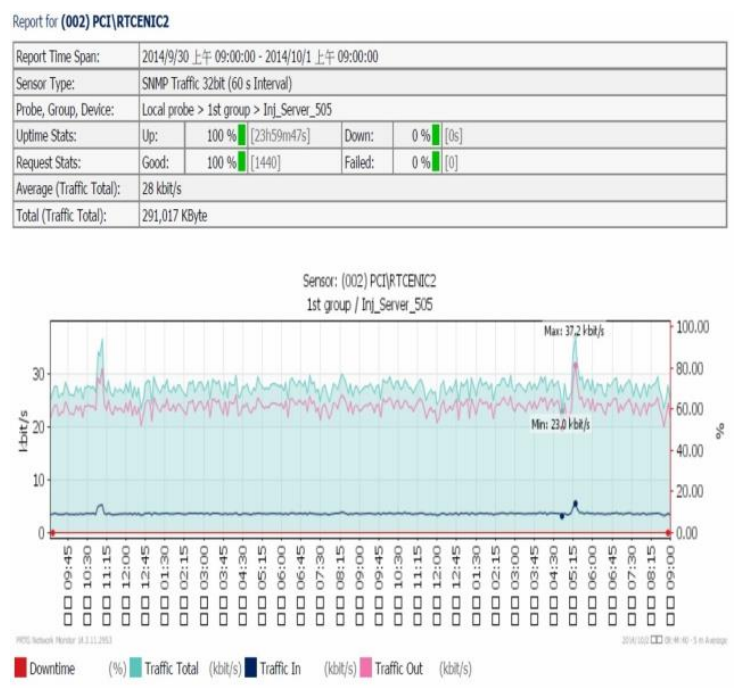

Fig. 10. Test results for the injection molding machine information retrieval agent under continuous execution for 24 hours for a single injection molding machine.

\section{EXPERIMENTAL RESULTS AND DISCUSSION}

Figure 10 depicts the test results for the injection molding machine information retrieval agent in the case of continuous execution of a single injection molding machine for 24 hours. The infrastructure of the Ethernet transmission is based on a 10/100M bps transmission bandwidth. As shown in the test results, the data transmission was $100 \%$ in the 24 hour interval with no data missing. It can be seen that the average network flow traffic transferred by this single injection molding machine was $28 \mathrm{kbits} / \mathrm{s}$. The total traffic was 291,017 Kbyte in 24 hours, which shows that the implemented production information retrieval agent executed well. As shown in the figure, there was a peak of transmission flow at $05: 15$, which described the data purge and warehousing of the injection molding machine controllers. The results indicate that the maximum transmission flow can be $37.2 \mathrm{Kbits} / \mathrm{s}$ at approximately 23.0 Kbits/s. In conclusion, the testing results showed that the proposed injection molding machine information retrieval agent can work well with the designed data encryption mechanism and that the transferred production information is stable.

\section{CONCLUSIONSS \&S FURTHERS RESEARCH}

In this paper, it has been presented a working definition for Smart Injection Molding and Molds 4.0. Furthermore, a set of general recommendations in terms of sensors, data acquisition system, and signals monitoring have been provided to support the construction of instrumented Smart Molds. Finally, an A- CPS SOA Architecture for Smart Injection Molding has been introduced to increase the competitiveness of the injection molding industry in preparation for the development of cyberphysical production system at the heart of Smart Factories in the emerging Industry 4.0 . 
In future works, the system will be extrapolated to other industrial scenarios, such as the automotive and consumer goods sectors in order to fully understand the impact of Smart Injection Molding in the evolution of the plastic processing industry. The final-outcome will be the generalization of the concept of Molds 4.0 in the manufacturing practice, towards the implementation of instrumented molds and the proposed A-CPS SOA Architecture in other processes as well.

\section{REFERENCES}

1. M. Sameer, K. Muztoba, D. Romero, T. Wuest. "Smart Manufacturing: Characteristics, Technologies and Enabling Factors". Proceedings of the Institution of Mechanical Engineers, Part B: Journal of Engineering Manufacture, DOI: 10.1177/0954405417736547, 2017.

2. A. Kusiak. "Smart Manufacturing Must Embrace Big Data". Nature, 544(7648):23-25, 2017

3. M. Tanli, Y. Jiang, X. Wang, Y. Wang, R. Peng. "Research on Global Structure for Digital Quality Testing Based on Manufacturing Factory". 2nd Int'l. Conference on Electrical, Automation and Mechanical Engineering (EAME 2017), DOI: 10.2991/eame-17.2017.42, 2017.

4. K.S. Wang. "Towards Zero-Defect Manufacturing (ZDM) - A Data Mining Approach". Adances in Manufacturing, 1(1):6274, 2013.

5. H. Lee, K. Ryu, Y. Cho. "A Framework of a Smart Injection Molding System Based on Real-time Data". Procedia Manufacturing, Vol. 11, pp. 1004-1011, 2017.

6. S. Kenig, A. Ben-David, M. Omer, A. Sadeh. "Control of Properties in Injection Molding by Neural Networks". Engineering Applications of Artificial Intelligence, 14(6):819 823, 2001.

7. M. Chalk. "Smart Moulds for Cost Effective Components". Reinforced Plastics, (47)10:30-34, 2003.

8. M.P. Papazoglou. "Web Services \& SOA: Principles and Technology". Pearson India, 2nd. Edition, 2015.

9. J.V. Abellan-Nebot, F. Romero Subirón. "A Review of Machining Monitoring Systems based on Artificial Intelligence Process Models". Int'l J. of Advanced Manufacturing Technology, 47(1-4):237-257, 2010.

10. P. Estrada, H.R. Siller, E. Vázquez, C.A. Rodríguez, O. Martínez- Romero, R. Corona. "Micro-Injection Moulding of Polymer Locking Ligation Systems". Procedia CIRP, Vol. 49, pp. 1-7, 2016

11. J. Lee, B. Bagheri, H.A. Kao. "Recent Advances and Trends of Cyber- Physical Systems and Big Data Analytics in Industrial Informatics". 12th IEEE Int'l. Conference on Industrial Informatics (INDIN), pp. 1-6, 2014.

12. M. Hermann, T. Pentek, B. Otto. "Design Principles for Industry 4.0 Scenarios". 49th IEEE Hawaii Int'1. Conference on System Sciences, pp. 3928-3937, 2016

13. R. Goncalves, D. Romero, A. Grilo. "Factories of the Future: Challenges and Leading Innovations in Intelligent Manufacturing". Int'l. J. of Computer Integrated Manufacturing, 30(1):4-14, 2017.

14. .0 Maturity Index - Managing the Digital Transformation of Companies". Acatech Study, 2017.

15. D. Powell, D. Romero, P. Gaiardelli, C. Cimini, S. Cavalieri. "Towards Digital Lean Cyber-Physical Production Systems: Industry 4.0 Technologies as Enablers of Leaner Production". IFIP, AICT 536, Part II, Springer, pp. 353-362, 2018.

16. P. Angulo, C.C. Guzmán, G. Jiménez, D. Romero. “A Service Oriented Architecture and its ICT-Infrastructure to support Eco-Eficiency Performance Monitoring in Manufacturing Enterprises". Int'l. J. of Computer Integrated Manufacturing, 30(1):202-214, 2017.

17. T. Wuest, T. Schmidt, W. Wei, D. Romero. "Towards (Proactive Intelligent Products". Int'l. J. of Product Lifecycle Management, 11(2):154-189, 2018.

18. R.J. Rabelo, L.M. Camarinha-Matos. "Negotiation in MultiAgent based Dynamic Scheduling”. Robotics \& Computer Integrated Manufacturing, 11(4):303-310, 1994.

19. G. Huang, D. Sheldon. "A Tandem Architecture for
Cooperating Knowledge-Based Concurrent Engineering". Engineering with Computers, 10(2):112-123, 1994.

20. H. Sneed, R. Majnar. "A Case Study in Software Wrapping”. IEEE Int'l. Conference on Software Maintenance, DOI: 10.1109/ ICSM.1998.738496, 1998.

21. H. Van Brussel, J. Wyns, P. Valckenaers, L. Bongaerts, P. Peeters. "Reference Architecture for Holonic Manufacturing Systems: PROSA". Computers in Industry, 37(3):255-274 1998.

22. P. Leitão. "Agent-based Distributed Manufacturing Control: A State-of- the-Art Survey". Engineering Applications of Artificial Intelligence, 22(7):979-991, 2009.

23. R. Zhong, X. Xu, E. Klotz, S. Newman. "Intelligent Manufacturing in the Context of Industry 4.0: A Review". Engineering, 3(5):616-630, 2017

24. R.J. Rabelo, D. Romero, S.P. Zambiasi. "Softbots Supporting the Operator 4.0 at Smart Factory Environments". IFIP, AICT 536, Part II, Springer, pp. 456-464, 2018.

25. FIPA ACL, http://www.fipa.org/repository/aclspecs.htm

26. M. Ketel. "Integration of Software Agent Technologies and Web Services". $3^{\text {rd }}$ Int'l. Multi-Conference on Society, Cybernetics and Informatics (IMSCI), 2009.

27. A. Micsik, P. Pallinger, A. Klein. "SOAP based Message Transport for the JADE Multiagent Platform". $8^{\text {th }}$ Int'l.Conference on Autonomous Agents and Multiagent Systems (AAMAS), pp. 101-104, 2009.

28. ISO 9506 https://www.iso.org/obp/ui/\#iso:std:iso:9506:-1:ed-2:v1:e

29. X. Lu, L. Khim. "A Statistical Experimental Study of the Injection Molding of Optical Lenses". Journal of Materials Processing Technology, 113(1):189-195, 2001.

30. B. Ozcelik, T. Erzurumlu. "Comparison of the Warpage Optimization in the Plastic Injection Molding using ANOVA, Neural Network Model and Genetic Algorithm”. Journal of Materials Processing Technology, (3):437-445, 2006

31. T. Erzurumlu, H. Oktem. "Comparison of Response Surface Model with Neural Network in Determining the Surface Quality of Moulded Parts”. Materials \& Design, 28(2):459 465, 2007.

32. C. Özek, Y.H. Çelık. "Calculating Molding Parameters in Plastic Injection Molds with ANN and Developing Software". Materials and Manufacturing Processes, 27(2):160-168, 2012.

33. S. Pattnaik, D. Karunakar, P. Jha. "Application of Computer Simulation for Finding Optimum Gate Location in Plastic Injection Moulding Process". Int'l. J. of Advanced Engineering Research and Studies, Vol. 1., pp. 159-161, 2012

34. S. Taghizadeh, A. Özdemir, O. Uluer. "Warpage Prediction in Plastic Injection Molded Part using Artificial Neural Network". Iranian Journal of Science and Technology, Transactions of Mechanical Engineering, 37(2):149-160, 2013.

35. R.X. Gao. "Online Product Quality Monitoring through Inprocess Measurement". CIRP Annals-Manufacturing Technology, 63(1):493- 496, 2014.

36. W.s Chen,s H.s Pen,s C.s Shu.s "Ans Integrateds Parameters Optimizations Systems fors MIMOs Plastics Injections Moldings usings Softs Computing”.s Int'l.s J.s ofs Advanceds Manufacturings Technology,s 73(9):1465-1474,s 2014.

37. J.s Nam.s "Injection-mouldeds Lenss forms Errors Predictions usings Cavitys Pressures ands Temperatures Signalss baseds ons Kfolds Crosss Validation".s Proceedingss ofs thes Institutions ofs Mechanicals Engineers,s Parts B:s Journals ofs Engineerings Manufacture,s DOI:s 10.1177/0954405416654421,s 2016.

38. S.s Atluru,s A.s Deshpande,s S.s Huang,s J.s Snyder.s " Smarts Machines Supervisorys System:s Concept,s Definitions ands Application".s 62nds Conferences ofs thes Societys fors Machinerys Failures Preventions Technology,s pp.s 6-8,s 2008.

39. S.s Atluru,s S.s Huang,s J.s Snyder.s "As Smarts Machines Supervisorys Systems Framework".s Int'l.s J.s ofs Advanceds Manufacturings Technology,s 58(5-s 8):563-572,s 2012. 